\title{
Surface Charge Control of Quantum Dot Blinking
}

\author{
Steven D. Quinn, Aidan Rafferty, Emma Dick, Michael J. Morten, Fraser J. Kettles, Chloe Knox, \\ Mark Murrie,* and Steven W. Magennis* \\ WestCHEM, School of Chemistry, University of Glasgow, Joseph Black Building, University Avenue, Glasgow G12 8QQ U.K.

\section{Supporting Information}

ABSTRACT: A characteristic property of colloidal semiconductor nanocrystal quantum dots (QDs) is their emission intermittency. Although a unifying theory of QD photoprocesses remains elusive, the importance of charged states is clear. We now report a new approach to directly study the role of surface charge on QD emission by adding metal ions to individual, core-only QDs immobilized in aqueous solution in an agarose gel. The CdTe QDs show very stable emission in the absence of metal ions but a dramatic and reversible increase in blinking due to the presence of trivalent metal ions. Our results support a charge-separation model, in which the major blinking pathway is the surface trapping of electrons; transiently bound metal ions close to the QD surface enhance this process.

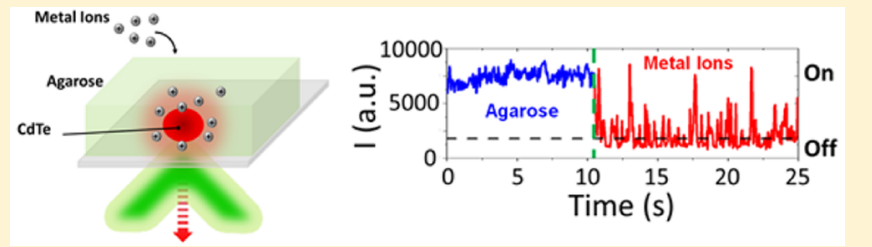

\section{INTRODUCTION}

Photoluminescence (PL) intermittency, also termed blinking, is a universal feature of emitters ranging from small molecular fluorophores, ${ }^{1,2}$ fluorescent proteins, ${ }^{3}$ and conjugated polymers ${ }^{4}$ to nanoscale emitters such as semiconductor quantum dots $(\mathrm{QDs})^{5}$ and diamond nanocrystals. ${ }^{6}$ Until recently, the blinking of QDs was primarily seen as a disadvantage, especially for applications such as particle tracking ${ }^{7}$ and solar cells ${ }^{8}$ where long-lived dark states are unwanted. In contrast, the emergence of super-resolution imaging techniques that utilize the stochastic switching of fluorescence between "on" and "off" states has meant that blinking QDs are also highly desirable. ${ }^{9-11}$ The resistance to photobleaching, together with other desirable optical properties, gives blinking QDs a distinct advantage in such applications.

It is well established that the PL intensity for QDs randomly jumps between highly emissive "on" states and nonemissive "off' states under continuous excitation., ${ }^{5,13}$ Rather than displaying two-state on/off dynamics, the on states display a continuous distribution of emissive states. ${ }^{14}$ The distribution of on and off times span up to 6 orders of magnitude, corresponding to almost 9 orders of magnitude in probability density, which are generally assigned to power law kinetics. ${ }^{15,16}$ Many studies have also reported truncated power law behavior, ${ }^{17}$ though the exact form of on/off distributions is sensitive to the methods employed for data analysis. ${ }^{18}$ There is also recent evidence that the apparent power law distributions are due to a superposition of exponential functions. ${ }^{16}$

Therefore, in spite of nearly two decades of experimental studies of the photophysical and charge transport properties of QDs, the mechanism of blinking in QDs is still the subject of vigorous debate. ${ }^{13,16,19,20}$ It is possible that several different mechanisms are operating in parallel, depending on the particular sample, environment, and experimental conditions. Nevertheless, there has been an attempt to find a universal blinking mechanism. The first model that gained acceptance involved the long-time charging of a single $\mathrm{QD}$ core, with subsequent excited-state energy lost nonradiatively via Auger recombination. ${ }^{21,22}$ However, various studies have questioned this model. ${ }^{23-25}$ The recently developed multiple recombination centers (MRC) ${ }^{26-28}$ model invokes multiple surface hole traps with fluctuating trapping rates. This has been recently extended to include additional electron trapping pathways, ${ }^{16}$ in part to accommodate experimental evidence that QD blinking can be altered through surface or solution modifications. ${ }^{29-31}$ More recent experiments ${ }^{32,33}$ have confirmed the existence of delayed emission, ${ }^{34,35}$ which can only be explained by longlived charge-separated states; a modified version of the Auger quenching model was proposed, with a suggested assignment of the electron as the trapped carrier. ${ }^{32,33}$

One of the reasons that it has been so difficult to develop a detailed blinking mechanism for QDs is the difficulty in correlating bulk charge distributions with specific surface processes. Important previous studies have involved adjusting the bulk solution environment with a change in $\mathrm{pH}^{36}$ via electrochemical methods, ${ }^{37,38}$ by intraparticle charge transfer ${ }^{39}$ by altering the bulk matrix, ${ }^{40-42}$ by adding a shell ${ }^{30}$ or capping ligands, ${ }^{29,31}$ or by applying external electric fields. ${ }^{43}$ In this work, we describe a new experimental approach to directly probe the effect of surface-localized charge on the QD emission by immobilizing core-only QDs in agarose gel and adding metal ions in aqueous solution (Figure 1a). We attribute the blinking to the occupation of surface traps enabled by kinetically labile positive metal ions bound to the agarose close to the QD surface. Our data are consistent with a model in which blinking results from the surface trapping of electrons. ${ }^{32,33}$

Received: August 2, 2016

Revised: August 5, 2016

Published: August 8, 2016 
(a) Quantum Dots

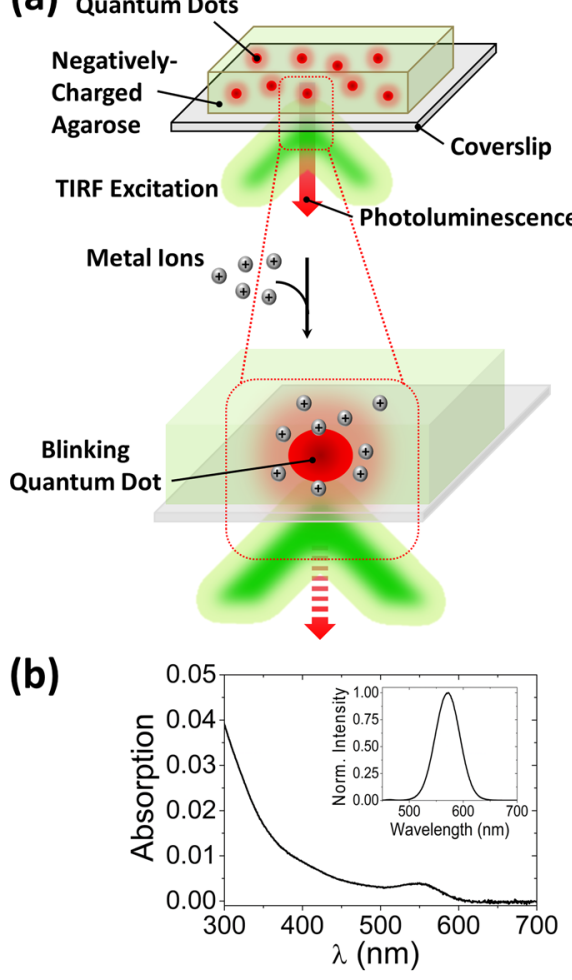

(c)

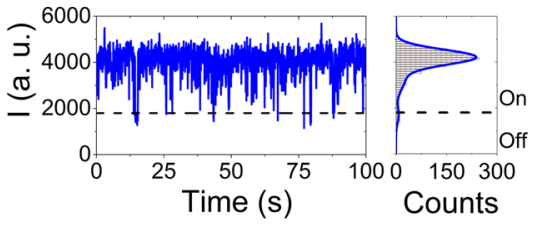

(d)

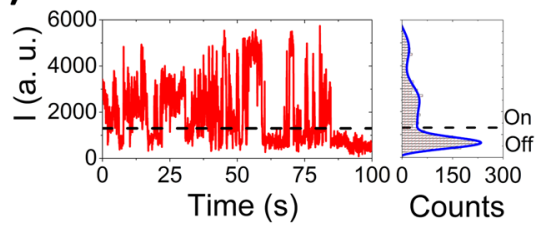

(e)

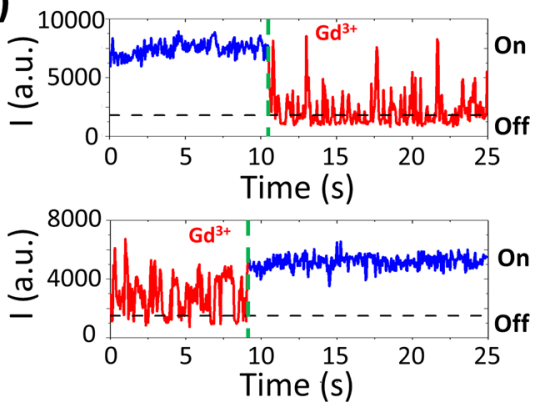

Figure 1. Surface charge control of CdTe quantum dot blinking. (a) Experimental design of single-particle imaging to visualize CdTe QDs immobilized within a $1 \%(\mathrm{w} / \mathrm{v})$ agarose gel at $\mathrm{pH} 8$ using wide-field o-TIRF microscopy (upper panel). The injection of trivalent ions, introduced as metal nitrates changes the emission properties (lower panel). (b) Absorption spectrum of CdTe QDs (25 nM) at pH 8; inset shows the fluorescence emission spectrum with $\lambda_{\text {exc }}=400 \mathrm{~nm}$. (c) Typical intensity trajectory of a single CdTe QD immobilized within $1 \%$ agarose gel at pH 8 with $532 \mathrm{~nm}$ excitation and (d) after injection of $10 \mu \mathrm{M} \mathrm{Gd}^{3+}$ ions. (e) The upper panel shows a single CdTe QD intensity trace in $1 \%$ agarose gel as Tris- $\mathrm{HCl}$ buffer containing $10 \mu \mathrm{M} \mathrm{Gd}^{3+}$ was injected at ca. $10 \mathrm{~s}$ (green dashed line). The lower panel demonstrates the reverse scenario where $\mathrm{Gd}^{3+}$ buffer was washed away by injecting $20 \mathrm{mM}$ Tris- $\mathrm{HCl}(\mathrm{pH} 8)$ buffer after ca. $10 \mathrm{~s}$.

\section{RESULTS AND DISCUSSION}

Hydrophilic CdTe QDs $\left(\lambda_{\mathrm{em}}=580 \mathrm{~nm}\right)$, which are capped with mercaptocarboxylic acids, were first characterized using ensemble spectroscopy in aqueous solution (Figure 1b). The QDs were stable at the concentrations and $\mathrm{pH}$ used for sample preparation (Figures S1 and S2). For single-particle experiments, the QDs were rapidly diluted to the $1-10 \mathrm{pM}$ level. Immobilization of QDs in agarose was achieved by adapting protocols used previously for immobilizing enzymes and proteins in an aqueous environment. ${ }^{44}$ Gels were prepared by adding 1-10 pM CdTe QDs to a buffered ( $\mathrm{pH} 8)$, molten $1 \%$ $(\mathrm{w} / \mathrm{v})$ agarose solution at $26-30{ }^{\circ} \mathrm{C}$. A small volume of the molten solution was added to a precleaned glass coverslip and allowed to cool and form a gel. The concentration of the QDs in solution was adjusted to give good surface density for singleparticle experiments. Emission trajectories of several hundred QDs in parallel were acquired using objective-type total internal reflection fluorescence (oTIRF) microscopy. All single-particle measurements were acquired with an excitation wavelength of $532 \mathrm{~nm}$ and excitation intensity of $130 \mathrm{~W} / \mathrm{cm}^{2}$.

The QD immobilization was found to be $\mathrm{pH}$ dependent. QDs added to agarose in $\mathrm{pH} 7$ buffer did not immobilize and were seen to be freely diffusing. Increasing the $\mathrm{pH}$ increased the number of fixed QDs, with $100 \%$ bound at $\mathrm{pH} 8$. We attribute this to Coulombic interactions of the QD with the agarose, which contains negatively charged substituents (particularly pyruvate and sulfate). ${ }^{45}$ Under dilute single-molecule conditions, the labile QD capping ligands can dissociate; bulk studies demonstrate slow aggregation (on a time scale of several minutes for a 10-fold dilution), which we attribute to ligand loss, as discussed previously (Figure S3). ${ }^{46}$ Addition of metal ions to such concentrated QD solutions leads to rapid aggregation (Figure S4 and Table S1). Such conditions have previously been used to study the effects of metal ions on QDs, ${ }^{47}$ but we believe that it is not possible to unambiguously assign diffraction limited spots to single QDs under such conditions. Our method of rapid dilution and immobilization in agarose avoids issues of aggregation.

Single-particle imaging of immobilized QDs was performed in the absence and presence of $\mathrm{M}\left(\mathrm{NO}_{3}\right)_{3}(\mathrm{aq})$, where $\mathrm{M}=\mathrm{Al}^{3+}$, $\mathrm{Y}^{3+}$, and $\mathrm{Gd}^{3+}$ (Figure 1a). We chose these metal ions because they are all tripositive, have large differences in ionic radius, and include both diamagnetic $\left(\mathrm{Al}^{3+}\right.$ and $\mathrm{Y}^{3+}$ ) and paramagnetic $\left(\mathrm{Gd}^{3+}\right)$ ions. In $1 \%(\mathrm{w} / \mathrm{v})$ agarose gel at $\mathrm{pH} 8$, the emission intensity of CdTe QDs displayed a notable lack of blinking observed on the time scales investigated (Figure 1c and Figure $\mathrm{S} 5)$. Core-shell QDs (CdSe/ZnS) have been studied in agarose previously, and the observed reduced blinking has been attributed to the negatively charged polysaccharide. ${ }^{48}$ In the presence of trivalent ions, however, we observed that the blinking of the CdTe QDs was greatly enhanced (Figure 1d). For example, for QDs incubated with $10 \mu \mathrm{M} \mathrm{Gd}^{3+}$, the majority of traces analyzed (70\%) displayed significantly higher levels of blinking, with only $3 \%$ in total remaining entirely photostable over the $100 \mathrm{~s}$ measurement time window. Notably, the predominant effect was to modulate the blink frequency rather 

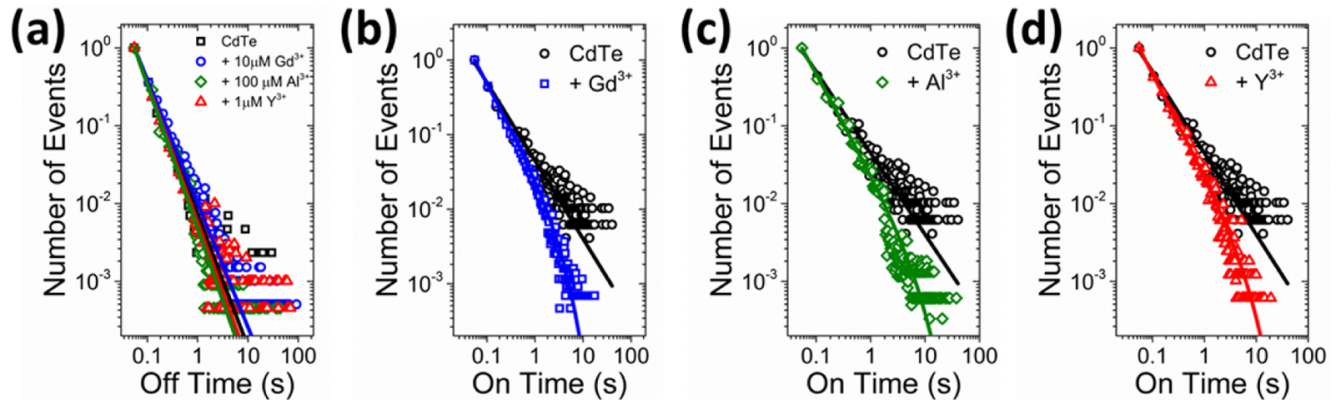

Figure 2. Statistics of on- and off-dwell times. (a) Normalized off-time histograms of CdTe immobilized within a 1\% (w/v) agarose gel (pH 8 ) in the absence (black) and presence of $10 \mu \mathrm{M} \mathrm{Gd}^{3+}$ (blue), $100 \mu \mathrm{M} \mathrm{Al}^{3+}$ (green), and $1 \mu \mathrm{M} \mathrm{Y}^{3+}$ (red) ions. The solid lines represent power law fits of the form $y=C t^{-\alpha}$, where $C$ is a constant, with similar exponents $(\alpha=1.70$ (black), 1.59 (blue), 1.83 (green), and 1.75 (red)). Normalized on-time histograms of immobilized CdTe in the absence (black) and presence of (b) $10 \mu \mathrm{M} \mathrm{Gd}^{3+}$ (blue), (c) $100 \mu \mathrm{M} \mathrm{Al}{ }^{3+}$ (green), and (d) $1 \mu \mathrm{M} \mathrm{Y} \mathrm{Y}^{3+}(\mathrm{red})$ are also shown. The solid black lines represent power law fits with $\alpha=1.04$. The solid blue, green, and red lines represent truncated power law fits $(y$ $=C t^{-\alpha} \mathrm{e}^{-\Gamma t}$, where $C$ is a constant) with similar exponents and truncation factors $\left(\alpha=1.20, \Gamma=0.30 \mathrm{~s}^{-1}\right.$ (blue); $\alpha=1.27, \Gamma=0.13 \mathrm{~s}^{-1}$ (green); $\alpha=$ $1.27, \Gamma=0.14 \mathrm{~s}^{-1}($ red $)$ ).

than the peak intensity. The effect of the trivalent ions appears to be instantaneous on our measurement time scale, since the blinking behavior was immediately altered as soon as they were injected into the sample (upper trace in Figure 1e). We found that this process could be reversed by flushing the QDs with buffer at $\mathrm{pH} 8$ (lower trace in Figure 1e). There is no change in $\mathrm{pH}$ upon adding the metal salts, ruling out this as a cause of the increased blinking.

To quantitatively analyze blinking, the on- and off-time distributions were obtained for immobilized QDs in the absence and presence of aqueous solutions of the trivalent ions. Blinking was analyzed by sampling the emission time trajectories using $50 \mathrm{~ms}$ time binning, and data were analyzed using algorithms in which the on- and off-states were distinguished using a threshold intensity level (see Supporting Information). For each measurement condition, dwell time histograms of on- and off-states were generated using data acquired from approximately 1200 QDs, and we were careful to avoid potential artifacts when analyzing on- and off-time distributions (see Supporting Information and Figures S6 and S7). ${ }^{18}$ All data were processed in an identical fashion to ensure a fair comparison between different measurement conditions. The off-time distributions in agarose before and after addition of metal ions displayed a very similar power-law dependence (Figure 2a and Table S2). In contrast, a substantial shortening of the on-time was observed upon addition of all three metal ions, and the distributions followed a truncated power law (Figure $2 \mathrm{~b}-\mathrm{d}$ and Table S2). Such truncated power laws have been observed many times, and most blinking models have incorporated this behavior. ${ }^{17}$

Although all three metal ions could induce similar blinking behavior, they required different concentrations to achieve the same effect. While $10 \mu \mathrm{M} \mathrm{Gd}^{3+}$ induced rapid blinking (Figure S8), identical experiments using $10 \mu \mathrm{M} \mathrm{Al}^{3+}$ only resulted in minor changes to the on-times (Figure S9). However, as the $\mathrm{Al}^{3+}$ concentration was raised to $100 \mu \mathrm{M}$, the on-times shortened with similar fitting parameters to those observed in the presence of $10 \mu \mathrm{M} \mathrm{Gd} \mathrm{d}^{3+}$ (Figure 2c). In contrast, the injection of $10 \mu \mathrm{M} \mathrm{Y}^{3+}$ induced QD displacement from the gel. Reducing the $\mathrm{Y}^{3+}$ concentration to $1 \mu \mathrm{M}$, however, resulted in immobilized QDs and similar blinking to $10 \mu \mathrm{M} \mathrm{Gd}^{3+}$ and 100 $\mu \mathrm{M} \mathrm{Al}^{3+}$ (Figure 2d).

To evaluate whether the presence of metal ions could alter the blinking statistics of QDs surrounded by a protective shell, the emission trajectories of single $\mathrm{CdSe} / \mathrm{ZnS}$ core-shell QDs immobilized within a $1 \%(\mathrm{w} / \mathrm{v})$ agarose gel were evaluated before and after addition of $\mathrm{Gd}^{3+}$. In contrast to the core-only CdTe QDs, no blinking enhancement was observed, and the off- and on-rates remained unchanged after $\mathrm{Gd}^{3+}$ addition (Figure S10), though we note that the core materials are different in these samples (CdTe vs $\mathrm{CdSe}$ ).

Our results have shown that trivalent metal ions are able to reversibly induce blinking, with very similar on-time distributions and little change in the off-time distribution (Table S2). It is clear that blinking depends on the metal, not the nitrate counterion; otherwise, we would not have a dependence of blinking on the salt concentration. The reversibility of the interaction in aqueous solution supports a labile ionic interaction rather than substitution of surface atoms. ${ }^{49}$ Furthermore, the difference in blinking observed for different metal ions is not due to variations in the bulk dielectric constant for a particular metal salt solution, as dielectric constant is primarily dictated by the charge of the cation. ${ }^{50}$

Instead, we attribute the blinking to the binding of the tripositive metal ions to the negatively charged agarose in the vicinity of the QD. In fact, the binding of trivalent metal ions to an anionic polysaccharide has been reported and ascribed to ionic interactions. ${ }^{51}$ Furthermore, the minimum concentration of trivalent metal ion needed to form gels varied widely in this same study, with an order of magnitude increase in concentration of $\mathrm{Al}^{3+}$ required, in comparison to $\mathrm{Y}^{3+}$ and heavier metals (including $\mathrm{Gd}^{3+}$ ). Similarly, our results suggest that the binding constants for interaction with the agarose also depend on the metal ion $\left(\mathrm{Y}^{3+}>\mathrm{Gd}^{3+}>\mathrm{Al}^{3+}\right)$. Although the metal ions are quite different in size, this is unlikely to be the cause of the different abilities to induce blinking (i.e., due to different ion-QD distances), since the effective sizes of hydrated trivalent metal ions (from $\mathrm{Al}^{3+}$ to $\mathrm{Ln}^{3+}$ ) are essentially identical. ${ }^{52}$ Since the $\mathrm{QD}$ binds to the anionic agarose, it might be expected that binding of a sufficient number of positive metal ions to the agarose would disrupt this interaction and lead to QD loss, which is exactly what we observed on addition of $10 \mu \mathrm{M} \mathrm{Y}^{3+}$.

\section{CONCLUSIONS}

In summary, we have developed a new approach to probe the effect of surface charge on the blinking of colloidal core-only QDs. Embedding the core-only CdTe QDs in agarose leads to 
very stable emission because the negatively charged agarose suppresses blinking by slowing electron trapping to surface sites (e.g., by reducing the rate of tunneling or by filling the electron trap sites). Similar explanations have been used to explain the effects of QD capping ligands. ${ }^{29,31}$ Trivalent metal ions can bind to the agarose leading to a reduction in the local negative charge in the vicinity of the QD and promoting the electron transfer pathway. The strong effect on the on-time distribution but negligible change in the off-time distribution suggests that the same process is responsible for the blinking dynamics with and without the metal ions, which supports the modified version of the long-time charging model. ${ }^{32}$ By studying a simple and reproducible system (core-only QD in an aqueous agarose system), we have been able to directly correlate blinking with QD surface charge. We believe that this work will contribute to the debate on emission intermittency and will encourage the use of similar approaches to directly probe the role of surface charge in this phenomenon.

\section{ASSOCIATED CONTENT}

\section{S Supporting Information}

The Supporting Information is available free of charge on the ACS Publications website at DOI: 10.1021/acs.jpcc.6b07779.

Experimental methods, Figures S1-S10, and Tables S1 and S2 (PDF)

\section{AUTHOR INFORMATION}

\section{Corresponding Authors}

*(S.W.M.) E-mail steven.magennis@glasgow.ac.uk.

*(M.M.) E-mail mark.murrie@glasgow.ac.uk.

\section{Notes}

The authors declare no competing financial interest.

\section{ACKNOWLEDGMENTS}

We thank the BBSRC for support of S.Q. (BB/K001957/1) and EPSRC for support of M.J.M. (EP/L027003/1) and F.K. (EP/I027203/1). We thank Prof. Lee Cronin (School of Chemistry, University of Glasgow, UK) for use of the dynamic light scattering facility.

\section{REFERENCES}

(1) Hoogenboom, J. P.; Hernando, J.; van Dijk, E. M. H. R.; van Hulst, N. F.; Garcia-Parajo, M. F. Power-Law Blinking in the Fluorescence of Single Organic Molecules. ChemPhysChem 2007, 8, 823-833.

(2) Haase, M.; Hubner, C. G.; Reuther, E.; Herrmann, A.; Mullen, K.; Basche, T. Exponential and Power-Law Kinetics in Single-Molecule Fluorescence Intermittency. J. Phys. Chem. B 2004, 108, 10445-10450.

(3) Dickson, R. M.; Cubitt, A. B.; Tsien, R. Y.; Moerner, W. E. On/ Off Blinking and Switching Behaviour of Single Molecules of Green Fluorescent Protein. Nature 1997, 388, 355-358.

(4) VandenBout, D. A.; Yip, W. T.; Hu, D. H.; Fu, D. K.; Swager, T. M.; Barbara, P. F. Discrete Intensity Jumps and Intramolecular Electronic Energy Transfer in the Spectroscopy of Single Conjugated Polymer Molecules. Science 1997, 277, 1074-1077.

(5) Nirmal, M.; Dabbousi, B. O.; Bawendi, M. G.; Macklin, J. J.; Trautman, J. K.; Harris, T. D.; Brus, L. E. Fluorescence Intermittency in Single Cadmium Selenide Nanocrystals. Nature 1996, 383, 802804.

(6) Bradac, C.; Gaebel, T.; Naidoo, N.; Sellars, M. J.; Twamley, J.; Brown, L. J.; Barnard, A. S.; Plakhotnik, T.; Zvyagin, A. V.; Rabeau, J. R. Observation and Control of Blinking Nitrogen-Vacancy Centres in Discrete Nanodiamonds. Nat. Nanotechnol. 2010, 5, 345-349.
(7) Lane, L. A.; Smith, A. M.; Lian, T.; Nie, S. Compact and BlinkingSuppressed Quantum Dots for Single-Particle Tracking in Live Cells. J. Phys. Chem. B 2014, 118, 14140-14147.

(8) Carey, G. H.; Abdelhady, A. L.; Ning, Z.; Thon, S. M.; Bakr, O. M.; Sargent, E. H. Colloidal Quantum Dot Solar Cells. Chem. Rev. 2015, 115, 12732-12763.

(9) Wang, Y.; Fruhwirth, G.; Cai, E.; Ng, T.; Selvin, P. R. 3D SuperResolution Imaging with Blinking Quantum Dots. Nano Lett. 2013, 13, 5233-5241.

(10) Watanabe, T. M.; Fukui, S.; Jin, T.; Fujii, F.; Yanagida, T. RealTime Nanoscopy by Using Blinking Enhanced Quantum Dots. Biophys. J. 2010, 99, L50-L52.

(11) Dertinger, T.; Colyer, R.; Iyer, G.; Weiss, S.; Enderlein, J. Fast, Background-Free, 3D Super-Resolution Optical Fluctuation Imaging (Sofi). Proc. Natl. Acad. Sci. U. S. A. 2009, 106, 22287-22292.

(12) Cichos, F.; von Borczyskowski, C.; Orrit, M. Power-Law Intermittency of Single Emitters. Curr. Opin. Colloid Interface Sci. 2007, $12,272-284$.

(13) Frantsuzov, P.; Kuno, M.; Janko, B.; Marcus, R. A. Universal Emission Intermittency in Quantum Dots, Nanorods and Nanowires. Nat. Phys. 2008, 4, 519-522.

(14) Zhang, K.; Chang, H. Y.; Fu, A. H.; Alivisatos, A. P.; Yang, H. Continuous Distribution of Emission States from Single CdSe/ZnS Quantum Dots. Nano Lett. 2006, 6, 843-847.

(15) Kuno, M.; Fromm, D. P.; Hamann, H. F.; Gallagher, A.; Nesbitt, D. J. "On" /"Off" Fluorescence Intermittency of Single Semiconductor Quantum Dots. J. Chem. Phys. 2001, 115, 1028-1040.

(16) Schmidt, R.; Krasselt, C.; Goehler, C.; von Borczyskowski, C. The Fluorescence Intermittency for Quantum Dots Is Not Power-Law Distributed: A Luminescence Intensity Resolved Approach. ACS Nano 2014, 8, 3506-3521.

(17) Cordones, A. A.; Leone, S. R. Mechanisms for Charge Trapping in Single Semiconductor Nanocrystals Probed by Fluorescence Blinking. Chem. Soc. Rev. 2013, 42, 3209-3221.

(18) Crouch, C. H.; Sauter, O.; Wu, X.; Purcell, R.; Querner, C.; Drndic, M.; Pelton, M. Facts and Artifacts in the Blinking Statistics of Semiconductor Nanocrystals. Nano Lett. 2010, 10, 1692-1698.

(19) Verberk, R.; van Oijen, A. M.; Orrit, M. Simple Model for the Power-Law Blinking of Single Semiconductor Nanocrystals. Phys. Rev. B: Condens. Matter Mater. Phys. 2002, 66, 233202.

(20) Jha, P. P.; Guyot-Sionnest, P. Trion Decay in Colloidal Quantum Dots. ACS Nano 2009, 3, 1011-1015.

(21) Efros, A. L.; Rosen, M. Random Telegraph Signal in the Photoluminescence Intensity of a Single Quantum Dot. Phys. Rev. Lett. 1997, 78, 1110-1113.

(22) Klimov, V. I.; Mikhailovsky, A. A.; McBranch, D. W.; Leatherdale, C. A.; Bawendi, M. G. Quantization of Multiparticle Auger Rates in Semiconductor Quantum Dots. Science 2000, 287, $1011-1013$.

(23) Rosen, S.; Schwartz, O.; Oron, D. Transient Fluorescence of the Off State in Blinking CdSe/CdS/ZnS Semiconductor Nanocrystals Is Not Governed by Auger Recombination. Phys. Rev. Lett. 2010, 104, 157404.

(24) Zhao, J.; Nair, G.; Fisher, B. R.; Bawendi, M. G. Challenge to the Charging Model of Semiconductor-Nanocrystal Fluorescence Intermittency from Off-State Quantum Yields and Multiexciton Blinking. Phys. Rev. Lett. 2010, 104, 157403.

(25) Cordones, A. A.; Bixby, T. J.; Leone, S. R. Direct Measurement of Off-State Trapping Rate Fluctuations in Single Quantum Dot Fluorescence. Nano Lett. 2011, 11, 3366-3369.

(26) Frantsuzov, P. A.; Volkan-Kacso, S.; Janko, B. Model of Fluorescence Intermittency of Single Colloidal Semiconductor Quantum Dots Using Multiple Recombination Centers. Phys. Rev. Lett. 2009, 103, 207402.

(27) Volkan-Kacso, S.; Frantsuzov, P. A.; Janko, B. Correlations between Subsequent Blinking Events in Single Quantum Dots. Nano Lett. 2010, 10, 2761-2765. 
(28) Frantsuzov, P. A.; Volkan-Kacso, S.; Janko, B. Universality of the Fluorescence Intermittency in Nanoscale Systems: Experiment and Theory. Nano Lett. 2013, 13, 402-408.

(29) Hohng, S.; Ha, T. Near-Complete Suppression of Quantum Dot Blinking in Ambient Conditions. J. Am. Chem. Soc. 2004, 126, 13241325.

(30) Chen, O.; et al. Compact High-Quality CdSe-CdS Core-Shell Nanocrystals with Narrow Emission Linewidths and Suppressed Blinking. Nat. Mater. 2013, 12, 445-451.

(31) He, H.; Qian, H.; Dong, C.; Wang, K.; Ren, J. Single Nonblinking CdTe Quantum Dots Synthesized in Aqueous Thiopropionic Acid. Angew. Chem., Int. Ed. 2006, 45, 7588-7591.

(32) Rabouw, F. T.; Kamp, M.; van Dijk-Moes, R. J. A.; Gamelin, D. R.; Koenderink, A. F.; Meijerink, A.; Vanmaekelbergh, D. Delayed Exciton Emission and Its Relation to Blinking in CdSe Quantum Dots. Nano Lett. 2015, 15, 7718-7725.

(33) Whitham, P. J.; Knowles, K. E.; Reid, P. J.; Gamelin, D. R. Photoluminescence Blinking and Reversible Electron Trapping in Copper-Doped CdSe Nanocrystals. Nano Lett. 2015, 15, 4045-4051.

(34) Sher, P. H.; Smith, J. M.; Dalgarno, P. A.; Warburton, R. J.; Chen, X.; Dobson, P. J.; Daniels, S. M.; Pickett, N. L.; O’Brien, P. Power Law Carrier Dynamics in Semiconductor Nanocrystals at Nanosecond Timescales. Appl. Phys. Lett. 2008, 92, 101111.

(35) Jones, M.; Lo, S. S.; Scholes, G. D. Quantitative Modeling of the Role of Surface Traps in CdSe/CdS/ZnS Nanocrystal Photoluminescence Decay Dynamics. Proc. Natl. Acad. Sci. U. S. A. 2009, 106, 3011-3016.

(36) Durisic, N.; Wiseman, P. W.; Gruetter, P.; Heyes, C. D. A Common Mechanism Underlies the Dark Fraction Formation and Fluorescence Blinking of Quantum Dots. ACS Nano 2009, 3, 11671175 .

(37) Jha, P. P.; Guyot-Sionnest, P. Electrochemical Switching of the Photoluminescence of Single Quantum Dots. J. Phys. Chem. C 2010, $114,21138-21141$.

(38) Galland, C.; Ghosh, Y.; Steinbrueck, A.; Sykora, M.; Hollingsworth, J. A.; Klimov, V. I.; Htoon, H. Two Types of Luminescence Blinking Revealed by Spectroelectrochemistry of Single Quantum Dots. Nature 2011, 479, 203-207.

(39) Tenne, R.; Teitelboim, A.; Rukenstein, P.; Dyshel, M.; Mokari, T.; Oron, D. Studying Quantum Dot Blinking through the Addition of an Engineered Inorganic Hole Trap. ACS Nano 2013, 7, 5084-5090.

(40) Issac, A.; von Borczyskowski, C.; Cichos, F. Correlation between Photoluminescence Intermittency of CdSe Quantum Dots and SelfTrapped States in Dielectric Media. Phys. Rev. B: Condens. Matter Mater. Phys. 2005, 71, 161302R.

(41) Issac, A.; Krasselt, C.; Cichos, F.; von Borczyskowski, C. Influence of the Dielectric Environment on the Photoluminescence Intermittency of CdSe Quantum Dots. ChemPhysChem 2012, 13, $3223-3230$.

(42) Mandal, A.; Nakayama, J.; Tamai, N.; Biju, V.; Isikawa, M. Optical and Dynamic Properties of Water-Soluble Highly Luminescent CdTe Quantum Dots. J. Phys. Chem. B 2007, 111, 12765-12771.

(43) Park, S.-J.; Link, S.; Miller, W. L.; Gesquiere, A.; Barbara, P. F. Effect of Electric Field on the Photoluminescence Intensity of Single CdSe Nanocrystals. Chem. Phys. 2007, 341, 169-174.

(44) Peterman, E. J. G.; Brasselet, S.; Moerner, W. E. The Fluorescence Dynamics of Single Molecules of Green Fluorescent Protein. J. Phys. Chem. A 1999, 103, 10553-10560.

(45) Serwer, P. Agarose Gels: Properties and Use for Electrophoresis. Electrophoresis 1983, 4, 375-382.

(46) Poderys, V.; Matulionyte, M.; Selskis, A.; Rotomskis, R. Interaction of Water-Soluble CdTe Quantum Dots with Bovine Serum Albumin. Nanoscale Res. Lett. 2010, 6, 9.

(47) Li, L.; Chen, Y.; Tian, G. J.; Akpe, V.; Xu, H.; Gan, L. M.; Skrtic, S.; Luo, Y.; Brismar, H.; Fu, Y. Reversible Modification of CdSe-CdS/ $\mathrm{ZnS}$ Quantum Dot Fluorescence by Surrounding $\mathrm{Ca}^{2+}$ Ions. J. Phys. Chem. C 2014, 118, 10424-10433.
(48) Ko, H. C.; Yuan, C. T.; Lin, S. H.; Tang, J. Blinking Suppression of Single Quantum Dots in Agarose Gel. Appl. Phys. Lett. 2010, 96, 012104.

(49) Sahu, A.; Kang, M. S.; Kompch, A.; Notthoff, C.; Wills, A. W.; Deng, D.; Winterer, M.; Frisbie, C. D.; Norris, D. J. Electronic Impurity Doping in CdSe Nanocrystals. Nano Lett. 2012, 12, 25872594.

(50) Hasted, J. B.; Ritson, D. M.; Collie, C. H. Dielectric Properties of Aqueous Ionic Solutions. Parts I and II. J. Chem. Phys. 1948, 16, 121.

(51) Quyen Thi le, N.; Okajima, M.; Mitsumata, T.; Kan, K.; Hang Thi, T.; Kaneko, T. Trivalent Metal-Mediated Gelation of Novel Supergiant Sulfated Polysaccharides Extracted from Aphanothece Stagnina. Colloid Polym. Sci. 2012, 290, 163-172.

(52) Kielland, J. Individual Activity Coefficients of Ions in Aqueous Solutions. J. Am. Chem. Soc. 1937, 59, 1675-1678. 